tungsebene zu diskutieren und zu entscheiden, ob und inwieweit sie sinnvoll gestaltet und umgesetzt werden können. Technische Sicherheitsmaßnahmen alleine reichen nicht aus, ebenso wichtig sind organisatorische Maßnahmen, die im Einklang mit der Gesamtstrategie der Institution stehen. Dabei sollte im Blick behalten werden, ob die Geschäftsprozesse und deren Schutzbedarf es zulassen, dass sich die zugehörigen Informationen mit ConsumerGeräten sicher, rechtskonform, wirtschaftlich und einfach handhabbar verarbeiten lassen.

Das Überblickspapier zu Consumerisation und BYOD ist auf der Webseite des BSI abrufbar: https://www.bsi.bund.de/DE/Themen/ITGrundschutz/Ueberblickspapiere/Ueberblickspapiere_node.htmI

\section{Andreas Könen neuer Vizepräsident des BSI}

Andreas Könen (51), Diplom-Mathematiker, hat seit 1. Januar 2013 die Position des Vizepräsidenten des Bundesamtes für Sicherheit in der Informationstechnik (BSI) inne und übernimmt damit das Amt seines Vorgängers Horst Flätgen.

Der neuen Position gingen verschiedene Stationen innerhalb des BSI voraus. So agierte Könen zuletzt als Leiter der Abteilung "Beratung und Koordination“ und übernahm in den Jahren zuvor die Leitungen der Fachbereiche "Koordination und Steuerung" sowie "Sicherheit in Anwendungen und Kritischen Infrastrukturen". 2006 trat Könen als Leiter des Leitungsstabes seinen Dienst beim BSI an. Von 1988 bis 2006 war er auf dem Gebiet der Informationssicherheit in der Bundesverwaltung tätig.

\section{EU-Pilotprojekt gegen Botnetze}

Am 01.02.2013 starteten die Arbeiten an einem europäischen Advanced Cyber Defense Center (ACDC). Partner aus 14 europäischen Staaten haben sich zusammengefunden, um gemeinsam gegen Botnetze vorzugehen.

Kern des EU-Pilotprojekts ist der Aufbau einer zentralen Datenbank mit Informationen über das Verhalten von schädlichen Programmen sowie die Ausführung koordinierter Gegenmaßnahmen durch die Mitgliedsstaaten. Im Rahmen des Projekts erfragt das Malware-Analyse-Team des Fraunhofer FKIE in Wachtberg und Bonn deutsche Erwartungen an ein Abwehrzentrum. Daneben entwickelt es Sensoren und bringt seine Kompetenzen in der Erkennung von Botnetzen und ihrer Verbreitung in das Abwehrzentrum ein.

Weitere Informationen auf der Projektwebsite: http://ec.europa. eu/information_society/apps/projects/factsheet/index.cfm?project_ ref $=325188$

\section{FP-Tochterunternehmen gibt Zusammenarbeit mit Identdienstleister ID 8 bekannt}

Mit der Partnerschaft der ID 8 GmbH und der Mentana-Claimsoft, Tochterunternehmen der börsennotierten Francotyp-Postalia Holding AG (FP), verbinden sich zwei namhafte De-Mail Pioniere. Die ID 8 ist spezialisiert auf Identitätsprüfungen und verfügt über ein bundesweites nach Signaturgesetz zertifiziertes Logistiksystem.
Von der getroffenen Vereinbarung profitieren neue De-Mail Kunden, sie können mit der ID 8 einen Termin am Wunschort für die Identifikation vereinbaren. Auch der Software Spezialist Formware und die deutsche FP Vertriebstochter haben die Möglichkeit erhalten, bei ihren Kunden das De-Mail Ident Verfahren durchführen zu dürfen.

De-Mail, die elektronische Alternative zum papiergebundenen Briefverkehr, macht den vertraulichen, sicheren, verschlüsselten und nachweisbaren Versand und Empfang von Dokumenten und Nachrichten über das Internet möglich. Im Prinzip funktioniert das wie bei einer herkömmlichen E-Mail - allerdings über eine eindeutige Identifikation von Sender und Empfänger.

Dafür müssen sich De-Mail-Nutzer einmalig mit einem Ausweis identifizieren, um ihren persönlichen De-Mail-Account zu erhalten. Dank dieser Erstregistrierung sind sowohl Empfänger als auch Absender klar identifizierbar, die digitale Post wird rechtsverbindlich und ist zum Beispiel auch für den Schriftverkehr mit Behörden geeignet.

Die Identitätsfeststellung der Teilnehmer am De-Mail Verfahren ist gesetzlich verankert und eine zentraler Schritt im Prozess. Deshalb müssen die Ident-Dienstleister für den De-Mail Provider Mentana-Claimsoft zugelassen werden. Das erfolgt im Rahmen eines Verfahren durch das Bundesamt für Sicherheit in der Informationstechnik (BSI), das die Funktionalität, Interoperabilität und Sicherheit bestätigt. Ebenfalls werden die datenschutzrechtlichen Anforderungen durch den Bundesbeauftragten für den Datenschutz und die Informationsfreiheit (BfDI) bestätigt. Dieses Vorgehen schafft bei den Anwendern die notwendige Akzeptanz eines sicheren DeMail Verfahrens.

\section{Internet-Kennzahlen-System (IKS): Öffentliche Online-Plattform offiziell gestartet}

Das Institut für Internet-Sicherheit - if(is) der Westfälischen Hochschule in Gelsenkirchen hat am 05.02.2013 mit dem Internet-Kennzahlen-System (IKS) eine Online-Plattform gestartet, die zahlreiche Daten für die Analyse und Bewertung des Internets bereitstellt. Als öffentliches Informations- und Analyseinstrument richtet sich die Plattform an Unternehmen, Behörden und Medien.

Das IKS ist Ergebnis eines Forschungsprojekts, das durch das Bundesminsterium für Wirtschaft und Technologie gefördert wird. Ziel ist es, das komplexe Gebilde Internet transparenter zu machen. Dazu stellt ein Expertenteam um Projektleiter Michael Sparenberg sogennante "Internet-Kennzahlen“, Maßzahlen für Zustände und Eigenschaften des Internets, zur Verfügung. Besucher der IKS-Website erhalten umfangreiche Informationen über die Nutzung, Bedrohung, Verfügbarkeit und Leistungsfähigkeit des Internets am Standort Deutschland. So bietet die Plattform etwa Analysen zur Robustheit des Internets und zu aktuellen Bedrohung durch Schadcode auf Webseiten.

Die aufbereiteten Internet-Kennzahlen können akademischen und behördlichen Institutionen als Grundlage neuer wissenschaftlicher Analysen dienen. Darüber hinaus sind sie für Entscheidungsträger der Internet-Wirtschaft wichtig, um aktuelle und künftige Potenziale einschätzen zu können. „Die IKS-Plattform ist im Internet einzigartig. Sie bietet die Möglichkeit, bessere Geschäftsentscheidungen in Politik und Wirtschaft herbeizuführen und liefert außerdem aktuelle Informationen für die mediale Berichtserstat- 